\title{
Cerebro-Oculo-Facio-Skeletal Syndrome (COFS)
}

National Institute of Neurological Disorders and Stroke (NINDS)

\section{Source}

National Institute of Neurological Disorders and Stroke (NINDS). Cerebro-Oculo-FacioSkeletal Syndrome (COFS) Information Page.

Cerebro-oculo-facio-skeletal syndrome (COFS) is a pediatric, genetic, degenerative disorder that involves the brain and the spinal cord. It is characterized by craniofacial and skeletal abnormalities, severely reduced muscle tone, and impairment of reflexes. Symptoms may include large, low-set ears, small eyes, microcephaly (abnormal smallness of the head), micrognathia (abnormal smallness of the jaws), clenched fists, wide-set nipples, vision impairments, involuntary eye movements, and impaired cog nitive development, which can be moderate or severe. Respiratory infections are frequent. COFS is diagnosed at birth. Ultrasound technology can detect fetuses with COFS at an early stage of pregnancy, as the fetus moves very little, and some of the abnormalities result, in part, from lack of movement.

A small number of individuals with COFS have a mutation in the "ERCC6" gene and are more appropriately diagnosed as having Cockayne Syndrome Type II. Other individuals with COFS may have defects in the xeroderma pigmentosum genes "XPG" or "XPD." Still others who are diagnosed with COFS have no identifiable genetic defect and are presumably affected because of mutations in a distinct, as-yet-unknown gene.

NOTE: This disorder is not the same as Cohen's syndrome (cerebral obesity ocular skeletalsyndrome). 